Revista

\title{
Multi-Ensayos
}

Vol. 7, núm. 13

ISSN: 2412-3285

https://multiensayos.unan.edu.ni

DOI: https://doi.org/10.5377/multiensayos.v7i13.10751

\section{Impacto de la COVID-19 en la formación y práctica de valores del profesional de Enfermería}

\section{Impact of COVID-19 on the training and practice of values of Nursing professional}

Jennifer Wong Matos ${ }^{1}$

Gabriela Samillán Yncio²

Elba Huaman Lanazca ${ }^{3}$

Alisson Maryorith Limaylla Villanueva ${ }^{4}$

Recibido: 29 de octubre de 2020. Aceptado: 16 de Noviembre de 2020

\section{RESUMEN}

La pandemia de la COVID-19 ha ocasionado una crisis en los sistemas de cada país; especialmente en los estados en desarrollo como el Perú, generó graves consecuencias en el sector salud, que afectó no solo a las personas que reciben atención, sino también a los profesionales que se encuentran en primera línea, sobre todo al profesional de Enfermería quien se encarga de brindar el cuidado integral al paciente, familia y comunidad. El cuidado es la esencia de Enfermería, conjuga conocimientos, habilidades y una serie de valores personales y profesionales, que son inherentes al quehacer laboral y dependen de la formación del profesional. El presente ensayo pretende generar reflexión ante la crisis y convertirla en una oportunidad para mejorar la formación en valores del enfermero y fortalecer su identidad moral, teniendo en cuenta que estos son esenciales para la práctica del cuidado, más aún frente a una situación adversa.

Palabras clave: crisis; cuidado; formación en valores; práctica del cuidado.

1 Universidad Nacional Mayor de San Marcos. Correo electrónico: jennifer.wong@unmsm.edu.pe 2 Universidad Nacional Mayor de San Marcos. Correo electrónico: gsamillani@unmsm.edu.pe 3 Universidad Nacional Mayor de San Marcos. Correo electrónico: elba.huaman1@unmsm.edu.pe 1 Universidad Nacional Mayor de San Marcos. Correo electrónico: alisson.limaylla@unmsm.edu.pe (c) 2021 Revista Multi-Ensayos. 


\section{ABSTRACT}

The COVID-19 pandemic has caused a crisis in the systems of each country; especially in developing states such as Peru, it generated serious consequences in the health sector, which affected not only the people who receive care, but also the professionals who are in first line, particularly the Nursing professional who is responsible for providing comprehensive and direct care to the patient, family and community. Care is the essence of Nursing, it combines knowledge, skills and a series of personal and professional values, which are inherent to the job and depend on the training of the professional. This essay aims to generate reflection about this crisis and turn it into an opportunity to improve the nurse's training in values and strengthen their moral identity, taking into account that these are essential for the practice of care, even more when it is necessary to face an adverse situation.

Keywords: crisis; care; training in values; practice of care.

\section{INTRODUCCIÓN}

La población mundial afronta una crisis sociosanitaria y educativa ocasionada por la pandemia COVID-19; que además generó un panorama lamentable evidenciado por altas tasas de morbimortalidad, especialmente en población vulnerable (Organización Mundial de la Salud. [OMS], 2020). En el Perú, el incremento vertiginoso de los casos de COVID-19 tuvo un gran impacto en el sistema de salud, visibilizó la precariedad de los servicios sanitarios, escasez de equipos e insumos médicos y condiciones laborales poco dignas para los profesionales (Maguiña, 2020). Aunado al incremento de la demanda de atención y preexistencia de brechas en el acceso a los servicios de salud, se ha presentado situaciones que podrían originar una crisis de valores en los profesionales de la salud; más aún, en las enfermeras que se encargan del cuidado humanizado de los pacientes hospitalizados.

Por otro lado, en el ámbito educativo, el cambio de la enseñanza presencial a la modalidad remota suscitó una transición inesperada que fue impuesta por el gobierno frente al panorama epidemiológico (D. S. N ${ }^{\circ}$ 008-2020-SA, 2020). También evidenció conductas inapropiadas en el plano de la ética, como la copia en las pruebas y trabajos; problemas que las universidades y docentes necesitan enfrentar y revertir con una sólida formación en valores como: honestidad, confianza, respeto y responsabilidad; orientada a la integridad académica y ciudadana, en la transición del proceso enseñanza-aprendizaje en línea virtual. (Pontificia Universidad Católica de Chile, 2020)

Ante este escenario, aparecen nuevos desafíos para la Enfermería, como la redimensión del cuidado al brindar atención en una emergencia sanitaria y en el aseguramiento de la calidad educativa que se desarrolla bajo la modalidad no presencial. Ello invita a reflexionar sobre ¿cómo revertir la crisis en una oportunidad?, además, la urgencia de enfrentar los retos, como la proyección a la innovación, la diversidad y la pertinencia de los currículos. Frente a esta realidad, los valores se constituyen en un saber esencial para la práctica del profesional de Enfermería y así garantizar la calidad del cuidado; para lograrlo, es importante una formación integral basada en valores que prepare a los enfermeros ante diversas situaciones inesperadas como la pandemia. 


\section{DESARROLLO}

La pandemia ha afectado a los diversos sectores del país, especialmente al área de salud, donde ya existía un sistema deficiente, produciendo un impacto negativo en la satisfacción del usuario externo e interno de las instituciones prestadoras de servicios sanitarios. La magnitud de esta situación a nivel laboral ha generado múltiples dilemas éticos para los responsables de la atención; entre ellos, los profesionales de Enfermería, quienes se vieron en la necesidad de priorizar los cuidados inmediatos, según las expectativas de vida de las personas, el afrontar una serie de crisis situacionales puede cuestionar sus valores durante la práctica profesional.

Este nuevo siglo avizora la integración de la tecnología en todos los ámbitos laborales, particularmente en los servicios de salud, donde la enfermera ofrece sus cuidados y, a la vez, enfrenta problemas éticos como la desigualdad, la exclusión y la importancia de un trabajo digno. Los valores constituyen un imperativo en el desarrollo de su práctica profesional porque "cuidar a todo ser humano llama a un compromiso científico, filosófico y moral, hacia la protección de su dignidad y la conservación de su vida" (Juárez y García, 2009, p. 113).

En tal sentido, para el sector salud es de gran importancia que los trabajadores en general no solo compartan principios y valores, sino que los interioricen y muestren sus actitudes de manera coherente ante las distintas situaciones. El cuidado de Enfermería es un proceso de acción que requiere integrar los valores personales con los profesionales, los mismos que son explicitados en la relación terapéutica entre la enfermera-individuo-familia-comunidad, desde la entrega de información hasta la participación en los diferentes procesos complejos que implica la praxis profesional como: la promoción, prevención, tratamiento y recuperación de la salud para la conservación de la vida. Este proceso ratifica que los valores humanos articulados a los conocimientos y habilidades permitirá que la enfermera brinde un cuidado integral.

Frente al contexto actual, el profesional de Enfermería se encuentra en la primera línea de defensa contra esta pandemia, enfrentando problemas estructurales como la escasez de camas y equipos que no permiten igualdad en los derechos de la atención; a pesar de eso, asumen jornadas largas y agobiantes que les genera cansancio físico, mental y exceso de estrés. Esta situación repercute en su estado de salud y se evidencia en el incremento del ausentismo (abandono laboral) y menor rendimiento físico, limitando la cantidad de servicios brindados. Lo cual se interpreta por terceros como la omisión de valores y principios éticos en la práctica cotidiana.

Las exigencias éticas son fundamentales y los profesionales de Enfermería están comprometidos con ello desde que inician su proceso de formación; constituyen la guía de su práctica profesional y, a nivel individual, implica autocuidado y protección del núcleo familiar. Posibilidades que no son atendidas por quienes no anticipan la escasez y vulneran su dinamismo, disposición, ecuanimidad e iniciativa de autocuidado, limitando su actuar y estado óptimo de salud. Esta circunstancia crítica respecto a los valores, constituye un imperativo categórico y requiere ser atendida por las instancias correspondientes. 
La esencia de Enfermería es el cuidado del ser humano en el proceso de enfermedad, este cuidado va de la mano con los principios éticos universales como son: la beneficencia, autonomía, justicia y responsabilidad; por lo tanto, si estos principios no se practican en el quehacer diario, entonces esa esencia se perdería gradualmente, obteniendo un cuidado monótono y automático. Por otro lado, esas atenciones tienen que empezar por uno mismo, es decir, conocerse y valorarse para poder prestar servicios a otros seres humanos que, en algún momento, pueden caer enfermos. Asimismo, las enfermeras son profesionales autónomas y justifican cada intervención que realizan en beneficio de la persona y su familia (Rodríguez y Rodríguez, 2018).

Toda persona tiene la capacidad para poder discernir entre el bien y el mal, este proceso se va desarrollando a lo largo de los años, iniciando desde el nacimiento, en el cual los padres son los que transmiten y practican los valores en el hogar; de este modo, el niño va interiorizándolos hasta llegar a una edad en la cual ya puede diferenciarlos y organizarlos, y esto se evidencia en su actuar cotidiano. Por ende, la infancia es el momento crucial para sentar las bases de los valores que, posteriormente, servirán en el desarrollo y quehacer profesional de acuerdo con las normas de la sociedad.

La conceptualización de la educación de valores se considera "un proceso continuo que comienza con la atención y educación del niño en la familia y se extiende hasta la Universidad" (Rodríguez et. al, 2014, p. 193). La práctica y formación de valores constituye un tema trascendente en la educación superior; "la Universidad es un entorno cuyos valores incluyen capacidad de innovación, identificación y búsqueda constante de nuevos caminos para crear nuevas oportunidades" (Costa et. al, 2020). En consecuencia, la formación del profesional de Enfermería debe estar basado en una educación en valores, ya que el cuidado es multidimensional; es decir, abarca diversos aspectos como: el biológico, físico, mental, social, político, espiritual, entre otros.

La formación en valores en el personal de Enfermería es un proceso que humaniza y dispone lo social de la profesión; de esta manera, se desarrolla la personalidad del profesional con enfoque integral, gracias a la modelación del ejercicio profesional en el proceso enseñanza-aprendizaje, durante la vida universitaria, con el objetivo de favorecer el desarrollo y práctica adecuada en el futuro comportamiento profesional. Por esta razón, el ejemplo de modelo de profesión debe ser definido y debe tener un carácter pluridimensional (Rodríguez, 2017).

En el campo educativo, el proceso de enseñanza aprendizaje no puede paralizarse y se ha optado por la modalidad remota, pero la realidad requiere atender la necesidad social a través de planes de formación continua para los profesionales de salud con miradas al futuro, particularmente de los enfermeros, quienes se ven afectados en todos los aspectos como consecuencia de la pandemia. De igual manera, los docentes se encuentran en la primera línea de la educación, enfrentando problemas estructurales como la conectividad y la desigualdad, incluso con esas deficiencias, continúan siendo guías en el proceso de educación de los enfermeros para que avancen en su desarrollo profesional. "El docente no solo tiene la responsabilidad formadora en el orden científico, sino también con su ejemplo puede impartir una conducta ética, moral y humanista" (Pérez, 2015, p. 278). 
La modalidad presencial y virtual no son excluyentes, se pretende desarrollar aprendizajes cognitivos programados, vinculándolos al aspecto afectivo a través del trabajo colectivo, para desarrollar una formación ética, promoviendo la reflexión en los valores personales. Por ese motivo, el docente actúa como guía, porque integra los valores personales a la formación integral y humanista de los enfermeros, fortaleciendo el sentir, el pensar y el actuar frente a todo ser humano.

En esta línea de contar con profesionales competentes que muestren relaciones interpersonales sustentados en la ética, se requiere también estudiantes que no solo dominen técnicas digitales y clínicas, sino que adopten valores al trasmitir o crear conocimientos, que muestren actitudes y conductas apropiadas en su aprendizaje y en sus interacciones con pares, esencialmente durante el quehacer diario. Dicha circunstancia demanda la necesidad de analizar la esencia de la enseñanza, en términos de valores tal como lo afirmó Febres (2013), es un "proceso que va más allá de impartir conocimientos, conlleva al desarrollo, la socialización e inculturación de las personas, a través del cual se forman las capacidades físicas, intelectuales, psicológicas y morales, que destacan en sus actitudes y conductas". Es otro reto que se agrega a la transición de pasar de una modalidad presencial a una didáctica virtual, sin que las tecnologías sustituyan al docente, sino que fortalezcan la relación alumno-docente para la formación integral.

Entonces, el currículo universitario que incluye formación de valores, "no se limita a un proceso lineal ni homogéneo de transmisión de información" (Reyes et. al, 2019, p. 3), el desarrollo de esta competencia consiste en permanente interacción con las personas; por lo tanto, en un medio virtual, el docente es vital como agente socializador, creativo y motivador. Actualmente, se necesita contextualizar el proceso educativo en correspondencia con la misión que las universidades cumplen con la sociedad; asimismo, se requiere planificar el proceso educativo y gestionar la información de acuerdo a las experiencias en el quehacer diario de Enfermería. Ante las conductas éticas que demanda la educación a distancia, también se necesita implementar un modelo educativo que tenga como eje central la reflexión de los valores, como la esencia de la formación integral del profesional de Enfermería.

En el ámbito laboral, respecto a la atención de los pacientes infectados por la COVID-19, se observa que el sistema de salud es injusto con los profesionales en diferentes regiones del Perú. Se evidencia deficiencias en la implementación de equipos de protección personal, contratos sin beneficios o temporales y escasa flexibilidad laboral, ante un profesional infectado con el virus. En consecuencia, los profesionales son sometidos a tomar decisiones personales e introspectivas, que luego serán proyectados hacia sus familias de manera positiva o negativa. Es un panorama lamentable que produce una serie de sentimientos, temores, miedo, ansiedad y varias interrogantes y expresiones como: "Si me contagio ¿dónde viviré?, ¿dónde me aislaré?, tengo miedo de contagiar a mi familia, espero no tener síntomas, ..."; sin embargo, los profesionales continúan en la batalla, porque presentan una conciencia del valor a la vida bien marcado durante su trabajo, estando comprometidos con la protección de la salud integral y la vida de la persona.

Por un lado, la igualdad que implica respetar los derechos del ser humano, entre los cuales se encuentran el derecho a la vida y el de recibir atención médica cuando sea necesario. Por otro lado, la dignidad humana, crucial en todo el proceso de atención o cuidado, incluye un trato cálido, respetuoso, empático y confidencial, sin importar los antecedentes de la persona. Este último valor se asocia con el altruismo 
que está basado en la preocupación por el bienestar de las personas mediante el cuidado, compromiso, generosidad, respeto a su cultura, creencias y percepciones. Estos valores son los que brindan el carácter humanista a la profesión de Enfermería durante el proceso del cuidado integral.

Del mismo modo, el profesional de Enfermería, probablemente se encuentre en otra disyuntiva sobre la justicia hacia sí mismo, versus la justicia hacia el paciente, dilema frecuente en el ejercicio profesional, con lo que se tendría que apelar a otro valor mayor: la vida. Además, ambas partes están en su derecho de reclamar y abogar por su vida, provocando así, otro dilema; por lo tanto, el ejercicio profesional está inmerso en un proceso continuo y dinámico donde se identifican problemas, se reflexiona ante ello y se brinda alternativas de solución.

Esos valores son básicos y siguen perdurando en el tiempo, inherentes al quehacer de Enfermería; están asociados a determinadas actitudes y comportamientos, independientemente de la cultura del profesional que lo ponga en práctica (Martín, 2017). Esta deontología intrínseca influye en el profesional de Enfermería y se enfoca en la importancia de ejercer el bien en las acciones, asumiendo las situaciones como propias, con responsabilidad y empatía. Esta motivación interna asociada a los valores, lleva a las enfermeras a continuar sus funciones a pesar de la crisis, en beneficio de la persona, familia y comunidad (Kant, 2002).

Las instituciones prestadoras de los servicios de salud cumplen un papel importante y tienen una responsabilidad compartida para afrontar la pandemia de la COVID-19, promoviendo asistencia, diagnóstico y tratamiento a los pacientes; garantizando la seguridad de los trabajadores sanitarios que se encuentran en primera línea. Desde inicios de la pandemia, hubo cambios en el flujo de atención a los usuarios, implicando que el profesional de Enfermería se adapte al contexto, sin dejar de lado los valores y principios éticos, manteniendo la calidad de atención y siguiendo los protocolos establecidos, a pesar de ciertas falencias en el sistema sanitario.

Los profesionales de Enfermería están expuestos a enfrentar conflictos de carácter ético, debido a las responsabilidades propias asociadas al cuidado de las personas y el hecho de trabajar en un ámbito de salud más tecnificado y complejo, con aspectos éticos relacionados a los avances tecnológicos en los servicios de salud. Para tal fin, es necesario que los profesionales de Enfermería se capaciten continuamente en el uso y aplicación de herramientas tecnológicas para la atención de los usuarios, teniendo en cuenta criterios y valores éticos con una visión holística y humanista del cuidado de las personas, lo cual implica que la enfermera trate al ser humano con respeto y absoluta dignidad (Escobar y Cid, 2018).

Es evidente el uso y el desarrollo de las nuevas tecnologías en el quehacer diario; sin embargo, no debería influir en la relación enfermera-paciente durante la práctica del cuidado, ya que esta interacción permite evidenciar los valores profesionales, donde prevalece el carácter humanista; de la misma manera, estos valores influyen en la habilidad de resolver y priorizar las situaciones que ayuden a humanizar el cuidado y toda intervención que se realice con el paciente y su familia en cualquier contexto, tomando en cuenta sus necesidades y recursos. 


\section{CONCLUSIONES}

La crisis aún no ha cesado y el futuro es impredecible; la demanda de la calidad de los servicios de salud se mantiene evidente y el profesional de Enfermería exige comprensión y respeto a sus derechos humanos y al trabajo digno. De igual manera, como profesional humanístico, doblega esfuerzo en su apego a valores propios de su función social y responsabilidad profesional, integrando diversos valores durante el proceso de cuidado. Esta situación requiere ser prioridad en la agenda política de los gobernantes.

La pandemia ha generado la oportunidad de repensar la articulación ineludible de la práctica profesional con la formación integral del enfermero, ambas han sido impactadas en sus esferas valorativas, motivacionales y conductuales. Por tal motivo, los diferentes escenarios del proceso formativo cobran relevancia, como: los servicios de salud en todos los niveles de atención y ámbitos académicos. Este hecho demanda un diálogo entre los decisores políticos de salud y educación, con la finalidad de consensuar una estrategia nacional para enfrentar la repercusión de la pandemia, prestando especial atención a los valores en el marco del desarrollo humano.

La universidad ha gestionado la crisis gracias al personal docente, lo cual ha permitido continuar la educación de manera remota; sin embargo, se han presentado ciertas dificultades como la desigualdad y conectividad. Esta situación amerita la gestión de planes de estímulos para asegurar el valor de la equidad a través de la inclusión en el ámbito educativo.

Entre los retos de los docentes trasciende el hecho de centrar esfuerzos en la creatividad para el diseño e implementación de estrategias didácticas, tanto en pregrado como posgrado, que promuevan la autovaloración y coevaluación, el respeto al otro, la autocrítica y proyección al cambio, ofreciendo una educación caracterizada por el equilibrio entre el conocimiento científico-tecnológico y los valores que son la esencia de la formación integral.

La formación en valores del profesional de Enfermería debería fortalecerse en las instituciones educativas, en correspondencia con los cuatro pilares fundamentales que rigen la educación actual: aprender a conocer, aprender a hacer, aprender a vivir juntos y aprender a ser. Esto permitirá a dichos profesionales, planificar y ejecutar intervenciones con un enfoque holístico, además tendrá una mirada filosófica de la vida, durante el quehacer diario, donde prevalezca el compromiso, la empatía, el altruismo, el respeto a la igualdad y la dignidad humana.

\section{REFERENCIAS}

Costa, R., Mota Lino, M., Jatobá de Souza, A. I., Lorenzini, E., Manfrini Fernandes, G. C., Brehmer, L. C. de F., Vargas, M. A. de O., Honório Locks, M. O. y Gonçalves, N. (2020). Enseñanza de enfermería en tiempos de Covid-19: ¿Cómo reinventarse en este contexto? Texto contexto - Enfermería, 29. https://www.scielo.br/pdf/tce/v29/es_1980-265X-tce-29-e20200202.pdf

D. S. N008-2020-SA. Que declara en Emergencia Sanitaria a nivel nacional por el plazo de noventa (90) días calendario y dicta medidas de prevención y control del covid-19 Diario Oficial El Peruano (2020). http://busquedas.elperuano.pe/normaslegales/decreto-supremo-que-declara-en- 
emergencia-sanitaria-a-nivel-decreto-supremo-n-008-2020-sa-1863981-2/

Escobar-Castellanos, B y Cid-Henríquez, P. (2018). El cuidado de enfermería y la ética derivados del avance tecnológico en salud. Acta Bioethica, 24(1), 39-46. https://scielo.conicyt.cl/pdf/abioeth/ v24n1/1726-569X-abioeth-24-01-00039.pdf

Febres, N. (2013). Valores en el docente universitario: una exigencia en la actualidad. Revista Educación en Valores, 1(19). http://servicio.bc.uc.edu.ve/multidisciplinarias/educacion-en-valores/v1n19/art07. pdf

Juárez-Rodríguez, P. A. y García-Campos, M. L. (2009). La importancia del cuidado de enfermería. Revista de Enfermería del Instituto Mexicano del Seguro Social, 17(2), 113-115. https://www.medigraphic. com/pdfs/enfermeriaimss/eim-2009/eim092j.pdf

Kant, I. (2002). Fundamentación para una metafísica de las costumbres (Trad. R. R. Aramayo). Alianza Editorial. (Trabajo original publicado ca. 1785).

Maguiña Vargas, Ciro. (2020). Reflexiones sobre el COVID-19, el Colegio Médico del Perú y la Salud Pública. Acta Médica Peruana, 37(1), 8-10. https://dx.doi.org/10.35663/amp.2020.371.929

Martín Ferreres, L. (2017). La presencia de los valores en la práctica enfermería. [Tesis de Doctorado, Universidad Internacional de Catalunya]. https://www.tdx.cat/bitstream/handle/10803/456048/ Tesis\%20M\%C2\%AA\%20Luisa\%20Mart\%C3\%ADn\%20Ferreres.pdf?sequence=12isAllowed=y

Organización Mundial de la Salud. (11 de noviembre de 2020). Reporte de la situación del coronavirus (COVID-19), por país, territorio y área. https://coronavirus.jhu.edu/map.html

Pérez Caballero, M. D. (2015). Formación de valores en los profesionales de la Salud. Revista Cubana de Medicina, 54(4), 278-279. http://scielo.sld.cu/pdf/med/v54n4/med01415.pdf

Pontificia Universidad Católica de Chile (2020, 30 de abril). PUCC Los desafíos de la integridad académica frente a la educación a distancia. [Comunicado de prensa]. http://www.uc.cl/noticias/los-desafiosde-la-integridad-academica-frente-a-la-educacion-a-distancia/

Reyes Alamilla, O. I. y Hernández Romero, G. (2019). Identificación y práctica de valores en la formación universitaria. Revista Cubana de Educación Superior, 38(2). http://scielo.sld.cu/pdf/rces/v38n2/02574314-rces-38-02-e17.pdf

Rodríguez Abrahantes, T. N., Trujillo Rodríguez, Y., Del Castillo Salazar, D. y Martínez Espino, M. (2014). El enfoque axiológico o valorativo de las ciencias médicas. Edumecentro, 6(3), 193-206. http://scielo. sld.cu/pdf/edu/v6n3/edu14314.pdf

Rodríguez Abrahantes, T. N. y Rodríguez Abrahantes, A. (2018). Dimensión ética del cuidado de enfermería. Revista Cubana de Enfermería, 34(3). http://www.revenfermeria.sld.cu/index.php/enf/article/ view/2430/383

Rodríguez Herrera, A. (2017). Valores: utopía o necesidad para enfermeros. Revista Médica Electrónica, 39(1), 791-802. http://scielo.sld.cu/scielo.php?script=sci_arttext\&pid=S1684-18242017000700010\&lng $=$ es\&tlng=es. 